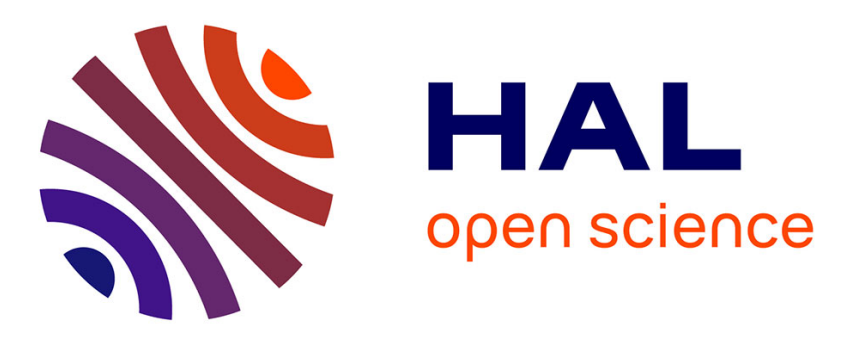

\title{
Explicitly distributed AOP using AWED
}

Luis Daniel Benavides Navarro, Mario Südholt, Wim Vanderperren, Bruno de Fraine, Davy Suvée

\section{To cite this version:}

Luis Daniel Benavides Navarro, Mario Südholt, Wim Vanderperren, Bruno de Fraine, Davy Suvée. Explicitly distributed AOP using AWED. 5th international conference on Aspect-Oriented Software Development, Mar 2006, Germany. pp.51-62, 10.1145/1119655.1119665 . hal-00486841

\section{HAL Id: hal-00486841 https://hal.science/hal-00486841}

Submitted on 26 May 2010

HAL is a multi-disciplinary open access archive for the deposit and dissemination of scientific research documents, whether they are published or not. The documents may come from teaching and research institutions in France or abroad, or from public or private research centers.
L'archive ouverte pluridisciplinaire HAL, est destinée au dépôt et à la diffusion de documents scientifiques de niveau recherche, publiés ou non, émanant des établissements d'enseignement et de recherche français ou étrangers, des laboratoires publics ou privés. 


\section{Explicitly distributed AOP using AWED}

\author{
Luis Daniel Benavides Navarro, \\ Mario Südholt \\ OBASCO project \\ École des Mines de Nantes/INRIA \\ 4 rue Alfred Kastler \\ 44307 Nantes cedex 3, France \\ \{lbenavid,sudholt\}@emn.fr
}

\author{
Wim Vanderperren, Bruno De Fraine, \\ Davy Suvée \\ System and Software Engineering Lab \\ Vrije Universiteit Brussel \\ Pleinlaan 2 \\ 1050 Brussels, Belgium \\ \{wvdperre,bdefrain,dsuvee\}@vub.ac.be
}

\begin{abstract}
Distribution-related concerns, such as data replication, often crosscut the business code of a distributed application. Currently such crosscutting concerns are frequently realized on top of distributed frameworks, such as EJBs, and initial AO support for the modularization of such crosscutting concerns, e.g., JBoss AOP and Spring AOP, has been proposed.

Based on an investigation of the implementation of replicated caches using JBoss Cache, we motivate that crosscutting concerns of distributed applications benefit from an aspect language for explicit distributed programming. We propose AWED, a new aspect language with explicit distributed programming mechanisms, which provides three contributions. First, remote pointcut constructors which are more general than those of previous related approaches, in particular, supporting remote sequences. Second, a notion of distributed advice with support for asynchronous and synchronous execution. Third, a notion of distributed aspects including models for the deployment, instantiation and state sharing of aspects. We show several concrete examples how AWED can be used to modularly implement and extend replicated cache implementations. Finally, we present a prototype implementation of AWED, which we have realized by extending JAsCo, a system providing dynamic aspects for Java.
\end{abstract}

\section{Categories and Subject Descriptors}

C.2 [Computer-communication networks]: Distributed Systems-Distributed applications; D.3.3 [Programming languages]: Language Constructs and Features

\section{General Terms}

Languages, Experimentation

${ }^{\dagger}$ This work has been supported by AOSD-Europe, the Eu-
ropean Network of Excellence in AOSD (aosd-europe.net).

Permission to make digital or hard copies of all or part of this work for personal or classroom use is granted without fee provided that copies are not made or distributed for profit or commercial advantage and that copies bear this notice and the full citation on the first page. To copy otherwise, to republish, to post on servers or to redistribute to lists, requires prior specific permission and/or a fee.

AOSD 06, March 20-24, 2006, Bonn, Germany

Copyright 2006 ACM 1-59593-300-X/06/03 ...\$5.00.

\section{Keywords}

Aspects, remote pointcuts, AWED, distributed execution, distributed language constructs.

\section{INTRODUCTION}

Distributed applications are inherently more complex to develop than sequential ones because of the additional requirements brought about by a partitioning of the software system across the network (e.g., handling of communication and synchronization between system components, network failures, management of load balancing, ...). Previous research has shown that traditional programming languages do not allow to separate well distribution concerns from standard functional concerns [34]. Web caching [28, 10] and unit testing of distributed applications [24], for instance, have been shown to be subject to serious crosscutting problems. Techniques developed in the field of Aspect-Oriented Software Development (AOSD) [22] should be useful to separate distribution concerns. However, despite its increasing popularity for sequential applications, relatively few AO approaches address the development of distributed software.

By now, a number of distributed middleware solutions have been developed that offer features for aspect-oriented development, such as JBoss AOP [1] and Spring AOP [3]. However, these solutions in essence apply a non-distributed $\mathrm{AO}$ system to an existing framework for distribution, such as J2EE. More specifically, they can only modify the distribution behavior of a base program by introduction (or removal) of distribution-related code expressed in terms of the underlying framework. Such approaches are therefore inherently limited to the framework's capabilities. In particular, they do not provide general support for explicit distribution in the aspect language or weaver technology, for instance, to support automatic deployment, state sharing and remote execution.

Few AO approaches, most notably D [34], JAC [25] and DJcutter [24], have support for explicit distribution in aspects. However, these approaches provide rather limited support for aspects which are triggered on conditions of programs running on remote hosts. Mechanisms to quantify over sets of hosts are lacking, and advice execution cannot be distributed over remote hosts. Sequence aspects, for instance, have recently been proven useful for system-level implementation of web caches [16], in particular protocol modifications, but none of the AO languages for distributed aspects support remote sequences. A larger set of distributed abstraction should support, in particular, a larger set of dis- 
tributed applications which may require diverse distributed implementation strategies.

To resolve these shortcomings, this paper introduces AWED, an aspect-oriented programming language with explicit support for distribution. AWED has three main characteristics. First, it offers remote pointcuts that can match events on remote hosts, including support for remote sequences. Second, it allows for distributed advice execution. Third, it provides a model of distributed aspects which addresses deployment, instantiation and data sharing issues. Furthermore, we present an implementation of AWED built on top of the dynamic AOP system JAsCo [30]. This implementation allows to dynamically apply distributed aspects and apply compiler optimizations such as just-in-time compilation and hot swapping. Finally, to motivate and illustrate our approach, we present a detailed analysis of crosscutting concerns in the context of the application server JBoss [2], using AWED, in particular, to address modularization issues of the JBoss Cache component.

This paper is structured as follows. Sec. 2 motivates crosscutting problems concerning replication and transaction concerns in JBoss cache. Sec. 3 presents the AWED aspect language. In Sec. 4 we show how AWED can be applied to different distribution problems, in particular distributed caching schemes. Sec. 5 introduces DJAsCo, our publicly-available prototype implementation of AWED on top of JAsCo. Sec 6 discusses related work and Sec. 7 gives a conclusion and presents future work.

\section{MOTIVATION: CACHE REPLICATION AND JBOSS CACHE}

As a motivating example we consider distribution problems arising in the context of the JBoss application server [2], which is built on J2EE, the Java-based middleware platform. Concretely, we consider replication in the JBoss Cache subsystems [7]. Replicated caches that provide a fast store close to client applications are a common solution to speed up distributed applications. The cache implementation of JBoss can be used to replicate data within a set of machines and thus ensures that all machines can access that data locally.

JBoss cache is a replicated transactional cache, i.e., the coherence of hte data in the caches forming a common cluster is ensured by guarding updates using transactions. A JBoss cache can either be configured to be local, in which case no data is replicated to other caches on other machines, or it can be global, which means that all changes are replicated to all the other caches (on all other machines) that are part of the cluster. Transactions in JBoss Cache are implemented using pessimistic locking. Once a transaction is finished on the local machine, a two phase commit protocol is initiated to replicate transactions. If all the caches can acquire the necessary local locks and make the modifications, a commit message is sent to finalize the transaction, otherwise the transaction is rolled back on all nodes.

In the following we focus on two modularization problems which JBoss Cache is subject to. (Note that we do not investigate if JBoss, which we consider a legacy application, could have been restructured to avoid these problems in the first place.) First, as we will analyze in detail below, the replication concern in JBoss Cache is crosscutting: it is scattered over large parts of its implementation and tangled with other scattered concerns. Second, modification of the

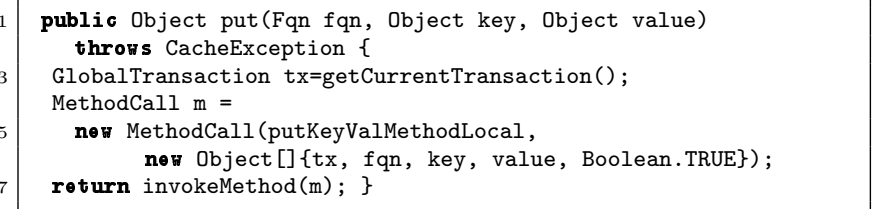

Figure 1: Low-level transaction handling in class TreeCache

standard behavior of JBoss Cache is also hindered by this crosscutting. It is, for instance, not possible to replicate specific data only in subsets of the caches from the cluster, once a cluster has been initialized.

JBoss Cache comes equipped with two AO-related mechanisms which could potentially be useful to overcome the modularization issues mentioned: an interception mechanism (package jboss . cache . interceptors) and JBossCacheAOP. With regard to the first mechanism, we show below that these interceptors do not resolve the crosscutting issues mentioned above. The second mechanism, JBossCacheAOP, is an Aspect-Oriented extension of JBoss Cache implemented using JBoss AOP, which allows to use JBoss Cache together with standard Java objects ("POJOs") in a transparent manner. JBossCacheAOP enables developers to simply put an object in a cache, and automatically handles the mapping of the object to the cache; the programmer can continue using POJOs as usual. This mechanism thus does not match our goals either: JBossCacheAOP is solely used to facilitate the use of JBoss Cache in an application but does not address modularization or extension of JBoss Cache's cache replication functionality.

Technically, the JBoss Cache implementation stores data in a tree data structure. The main tree data class is augmented with code to support a chain of interceptors, partially separating crosscutting concerns (e.g., replication, transactions, eviction policies and automatic access to backend data stores) in classes called interceptors. Each method invocation to a TreeCache object is then processed by the elements of the interceptor chain. The TreeCache class, however, is still subject to crosscutting by the code for replication and transactions. For instance, each low-level cache manipulation method, such as put has to get the transactional context, modify it if necessary and pass it along explicitly as shown in Figure 1, where the object of type MethodCall is used for replication purposes.

Figure 2 illustrates these crosscutting concerns by showing the scattering of replication and transaction code in class TreeCache (left diagram in the figure) in the interceptor package (right). Replication code is colored black in both subfigures, transaction code is marked dark gray and calls into the TreeCache method from the interceptor package are colored light gray. The figures clearly exhibit crosscutting of replication code, its tangling with transaction code and the interdependence between the TreeCache class and the interceptor package (the large number of calls from TreeCache into the interceptor package are not shown because they are frequently located near or part of the crosscutting code in the TreeCache class.)

More precisely, the TreeCache class includes 188 methods and consists of 1741 lines of code (LOC): the scattered code relevant for replication amounts to more than 196 LOC; 

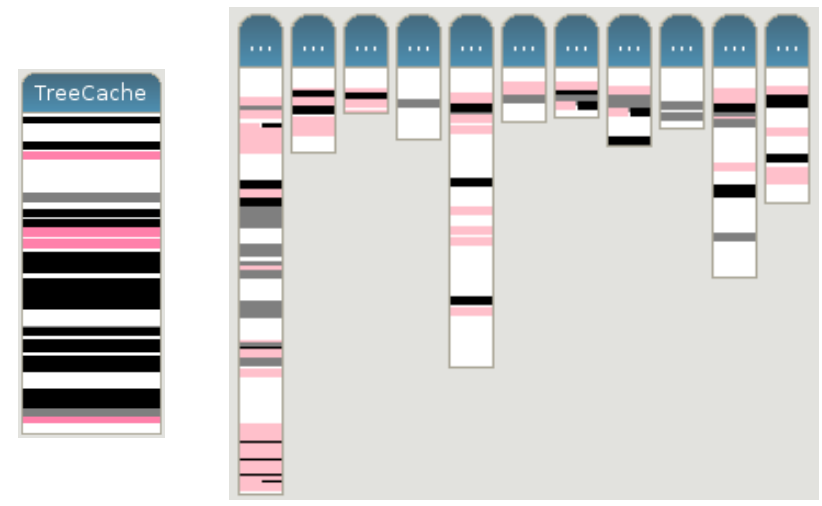

Figure 2: Crosscutting concerns in JBoss: class TreoCache (left), interceptor package (right)

the code for transactions accounts for more than 228 LOC. The situation for the interceptor framework is similar: it includes 9 classes consisting of 1263 LOC altogether; the (lower-bound) line counts for code relevant for replication, transactions and calls to TreeCache respectively are 30, 41, and 73. This provides strong evidence that the interceptor mechanism is not sufficient to solve the crosscutting problems.

Hence, understanding the replication and transactional behavior of the JBoss Cache implementation, which uses the interception mechanism, is far from trivial. Even simple modifications to the policies are difficult because of the crosscutting concerns. This applies, e.g., if the replication policy is to be changed to one where replication is done only when a cache is interested in some specific data and only within the subgroup of hosts that are also interested in the same data instead of replicating always between all members of a cluster. Finally, note that AspectJ-like languages are not appropriate in this context: as shown by Nishizawa et al. [24] they are subject to limitations, in particular, requiring inadequately complex aspect definitions in the context of distributed crosscutting functionalities.

\section{THE AWED LANGUAGE}

Modularization of crosscutting concerns for distributed applications using an aspect language, i.e., in terms of pointcut, advice and aspect abstractions, suggests support for the following issues: (i) a notion of remote pointcuts allowing to capture relationships between execution events occurring on different hosts, (ii) a notion of groups of hosts which can be referred to in pointcuts and manipulated in advice, (iii) execution of advice on different hosts in an asynchronous or synchronous way and (iv) flexible deployment, instantiation, and state sharing models for distributed aspects.

AWED provides such support through three key concepts at the language level. First, remote pointcuts, which enable matching of join points on remote hosts and include remote calls and remote cflow constructs (i.e., matching of nested calls over different machines). As an extension of previous approaches AWED supports remote regular sequences which smoothly integrate with JAsCo's stateful aspects [33] but also include features of other recent approaches for (nondistributed) regular sequence pointcuts $[14,15,16,4]$. Second, support for distributed advice: advice can be executed in an asynchronous or synchronous fashion on remote hosts and pointcuts can predicate on where advice is executed. Third, distributed aspects, which enable aspects to be configured using different deployment and instantiation options. Furthermore, aspect state can be shared flexibly among aspect instances on the one hand, as well as among sequence instances which are part of an aspect on the other hand.

\subsection{Syntax and semantics}

AWED's syntax is shown in Fig. 3 using EBNF formalism (i.e., square brackets express optionality; parentheses multiple occurrences, possibly none; terminal parentheses are enclosed in apostrophes).

\subsubsection{Pointcuts}

Pointcuts (which are generated by the non-terminal Pc) are basically built from call constructors (execution allows to denote the execution of the method body), field getters and setters, nested calls (cflow) and sequences of calls (nonterminal Seq).

AWED employs a model where, upon occurrence of a join point, pointcuts are evaluated on all hosts where the corresponding aspects are deployed. Pointcuts may then contain conditions about (groups of) hosts where join points originate (term host(Group)), i.e., where calls or field accesses occur. Furthermore, pointcuts may be defined in terms of where advice is executed (term on(Group)). Advice execution predicates may further specify a class implementing a selection strategy (using the term on(Group, Select)) which may, e.g., act as an additional filter or define an order in which the advice is executed on the different hosts. Groups are sets of hosts which may be constructed using the host specifications localhost, jphost and adr:port, which respectively denote the host where a pointcut matches, the host where the corresponding join point occurred and any specific host. Alternatively, groups may be referred to by name. (Named groups are managed dynamically within advice by adding and removing the host which an aspect is located on, see Sec. 3.1.2 below.)

Finally, pointcut definitions may extract information about the executing object (target) and arguments (args), and may test for equality of expressions (eq), the satisfaction of general conditions (if), and whether the pointcut lexically belongs to a given type (within). Pointcuts may also be combined using common logical operators.

As a first example, the following simple pointcut could be part of a replicated cache aspect:

call(void initCache()) \&\& host("adr1:port1")

Here, the pointcut matches calls to the cache's initCache method that originate from the host that has the specified address. The advice will be executed on any host where the aspect is deployed (possibly multiple ones) as there is no restriction on the advice execution host. The following example restricts the execution hosts to be different from the host where the joinpoint occurred:

pointcut putCache(Object key, Object o):

call $(*$ Cache.put(Object,Object))

\&\& !on(jphost) \&\& args (key, o)

Here, the pointcut matches calls to the cache's put operation on hosts other than the joinpoint host and binds the corresponding data items. (Note that in this case the clause !host(localhost) could replace !on(jphost) to achieve exactly the same effect of matching non-local joinpoints.) If 


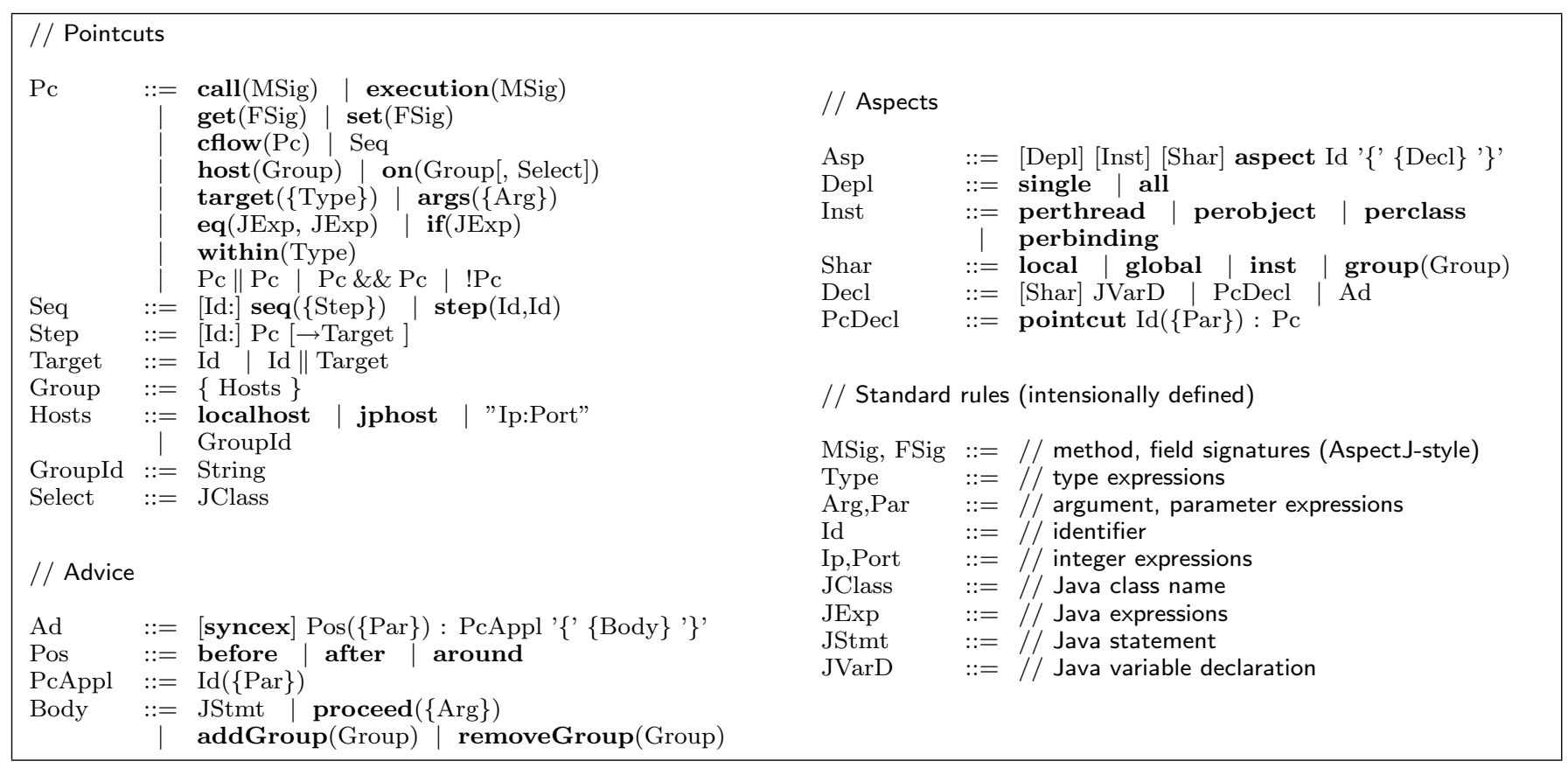

Figure 3: AWED language

the corresponding advice puts the item in the local cache, a condition on the aspect type (named, e.g., ReplCache), such as !within(ReplCache), can be used to avoid triggering the pointcut during the advice execution.

Sequences. Sequences (derived by the non-terminal Seq) are supported by two constructions on the pointcut level. First, the term [Id:] seq( $\{$ Step $\})$ allows to define sequences which may be named using an identifier and consist of a list of (potentially named) steps (non-terminal Step). A step may define the steps to be executed next (non-terminal Target). ${ }^{1}$ A sequence matches if the sequence is completed, i.e., if the current joinpoint matches the last step and previous joinpoints of the execution trace matched the previous steps in order. Second, the term step(seq,step) matches if the step named step of the sequence named seq matches. This allows advice to be triggered after a specific step within a sequence using a term of the form s: $\operatorname{seq}(\ldots 1: \operatorname{logout}() \ldots) \| \operatorname{step}($ Note that this last term matches the complete sequence besides the specified step; this can, if necessary, easily be ruled out. This sequence definition allows for a smooth integration of sequences and the finite-state aspects of non-distributed JAsCo.

To illustrate the use of sequence pointcuts, consider the following pointcut, which could be part of a simple cache replication protocol:

pointcut replPolicy (Cache c):

replS: seq(s1: initCache() \&\& $\operatorname{target}(\mathrm{c}) \rightarrow \mathrm{s} 3\|\mathrm{~s} 2\| \mathrm{s} 4$, s2: $\operatorname{cachePut}() \rightarrow \mathrm{s} 3\|\mathrm{~s} 2\| \mathrm{s} 4$, s3: stopCache ()$\rightarrow$ s1 s4: cacheInconsistency ()$)$

\footnotetext{
${ }^{1}$ Note that while our sequences obviously encode finite-state automata, many applications of regular structures, in particular communication protocols [16], are effectively sequencelike, i.e., of a one-dimensional directed structure, so that we decided to use the more intuitive terminology for AWED.
}

(Here, identifiers like initCache denote undefined pointcuts specifying corresponding call pointcuts.) The pointcut above defines a sequence of four steps. An initialization step which may be followed either by a put operation (s2), termination of the cache (s3) or an error step (s4). A put operation (s2) may be repeated, followed by a cache termination (s3) or result in a cache inconsistency (s4). After cache termination, the cache may be initialized once again. Finally, a cache inconsistency terminates the sequence (and may be reacted upon by advising the pointcut).

Note that the above definition does not enforce that the steps are taking in the context of the same cache. This is, however, simple to achieve by binding the targets of the different steps target(c), and use eq or if pointcuts to ensure the appropriate relationships at different steps in a sequence.

A step may be referred to in pointcuts and advice as exemplified in the following example which shows how to provide s, a. special pointcut for the second step in the previous sequence (and how to bind the variables used in that step) so that advice can later be attached to it:

pointcut putVal(Cache c, String key, Object o):

$\operatorname{step}(\mathrm{replS}, \mathrm{s} 2) \& \& \operatorname{target}(\mathrm{c}) \& \& \operatorname{args}(\mathrm{key}, \mathrm{o})$

\subsubsection{Advice}

Advice (non-terminal Ad) is mostly defined as in AspectJ: it specifies the position (Pos) where it is applied relative to the matched join point, a pointcut ( $\mathrm{PcAppl}$ ) which triggers the advice, a body (Body) constructed from Java statements, and the special statement proceed (which enables the original call to be executed).

In an environment where advice may be executed on other hosts (which is possible in AWED using the on pointcut specifier), the question of synchronization of advice execution with respect to the base application and other aspects arises. AWED supports two different synchronization modes for remote advice execution: by default remote advice is ex- 
ecuted asynchronously to the calling context. In this case synchronization, if necessary, has to be managed by hand. In contrast, syncex marks remote advice for synchronous execution. Local advice is always executed synchronously. The semantics of the proceed statement is "localized": the last around advice invokes the original behavior on the local host. The return value of the around advice is sent back to the original joinpoint host and processed in the regular around advice chain on that joinpoint host in case of a synchronous advice execution. Asynchronous advices are executed in parallel and there is thus no guarantee with respect to advice precedence. We have opted for this semantics because it provides for an intuitive yet efficient remote advice execution semantics.

Advice is also used to manage named groups of hosts: addGroup adds the current host to a given group, removeGroup allows to remove the current host from a group.

To give an example of basic advice functionality, the following advice definition is useful in the context of collaborating replicated caches:

around(String k, Object o): putCache $(\mathrm{k}, \mathrm{o})\{$

Object obj = getNewRemoteValue(k);

if $(\mathrm{obj} !=$ null $)\{\operatorname{proceed}(\mathrm{k}, \mathrm{obj}) ;\}$

else $\{\operatorname{proceed}(\mathrm{k}, \mathrm{o}) ;\}\}$

This advice first tests whether a new value is present remotely for a given key. If this is the case, the new value is stored in the cache.

\subsubsection{Aspects}

Aspects (non-terminal Asp) group a set of fields as well as pointcut and advice declarations. Aspects may be dynamically deployed (Depl) on all hosts (term all) or only the local one (term single).

Furthermore, aspects support four instantiation modes (Inst): similar to several other aspect languages, aspects may be instantiated per thread, per object, or per class. However, aspect instances may also be created for different sets of variable bindings arising from sequences (term perbinding) as introduced in $[16,4]$. In this last case, a new instance is created for each distinct set of bindings of the variables in the sequence, i.e., of the variables declared as arguments of a sequence pointcut or fields used in the sequence pointcut.

Finally, AWED allows distributed aspects of the same type to share local state (Shar): values of aspect fields may be shared among all aspects of the same type (term global), all instances of an aspect which have been created using the instantiation mechanisms introduced before (term inst), all aspects belonging to the same group (term group(Group)) or all aspects on the one host (term local; note that these possibly belong to different execution environments, such as JVMs). Sharing modifiers can be given for an aspect as a whole or individual fields (Decl), if both are given, the latter have priority.

\section{APPLICATIONS}

In this section, several applications of AWED are presented. We illustrate how replicated, cooperating distributed caches can be modularly implemented, and how distribution and execution clustering concerns can be introduced concisely into an existing non-distributed application.

\subsection{Caching revisited}

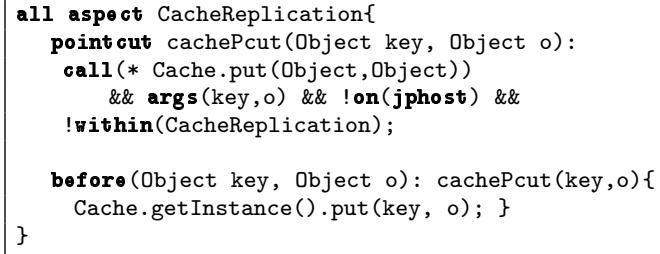

Figure 4: Cache replication as an aspect

In Sec. 2 we have presented distributed caching and, in particular, its support through the JBoss Cache OO framework, as a motivating example for crosscutting in distributed applications. Fig. 4 shows how an aspect for cache replication can be implemented using AWED which accounts for all places where cache elements are requested and replicated to all other caches in a cluster, i.e., an essential part of the functionality of replication within JBoss Cache's TreeCache class.

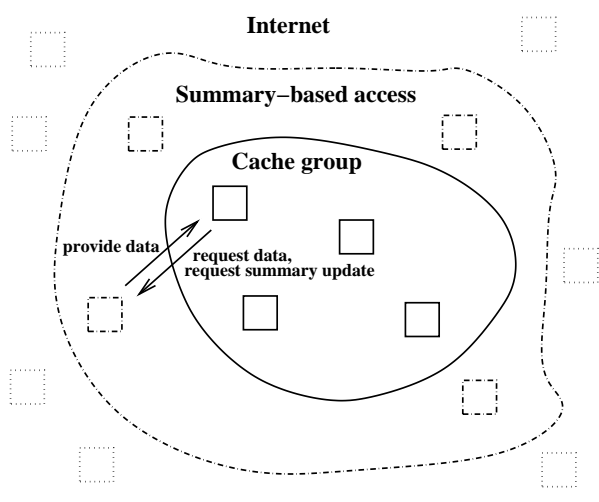

Figure 5: Adaptive cache behavior

The aspect declaration in line 1 indicates that the aspect will be distributed globally and that a singleton instance (AWED's default instantiation mode) is created on each host. The pointcut defined in lines $2-5$ matches calls putting elements in the cache; the term !on(jphost) limits advice execution to aspects which are not deployed on the host where the join point matched. The advice (lines 7-8) simply puts the element in the cache. As the pointcut assured that only aspects which are remote to the matching join point perform this advice, replication is thus achieved.

As a more intricate example, we consider an example of the large number of replication strategies for caches that use hierarchical, cooperative and adaptive caching strategies $[8$, 19]. Such strategies typically do not distribute data over whole clusters but replicate objects only to caches in the cluster that explicitly request them. Furthermore, cooperative behavior is useful, e.g., looking for a copy in neighboring caches before (slowly) accessing farther caches or a centralized server holding the master copy of the data at hand. This kind of behavior is not part of the current JBoss Cache specification and would be very difficult to graft on its implementation without the use of $\mathrm{AO}$ techniques due to the crosscutting problems of its replication code.

In the following we present the heart of a summary-based cooperative cache strategy using AWED. Summary-based caching strategies (see, e.g., [18]) use "summaries", i.e., 


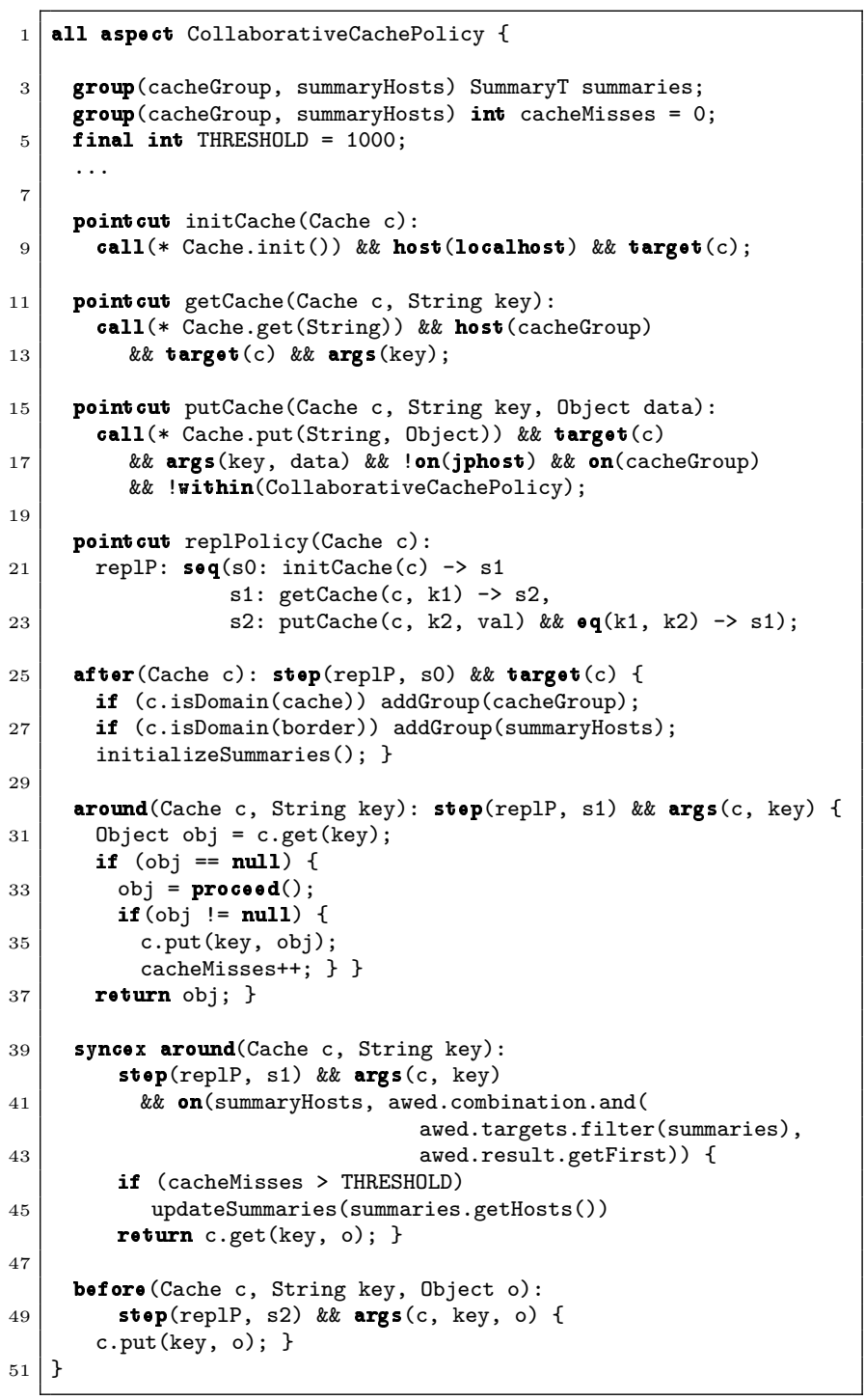

Figure 6: Aspect-based cooperative cache small digests of the cache contents of neighboring caches. The summaries can be used to test whether a cache contains a value with high probability. They can therefore be used to guide the decision which neighboring caches to contact and thus reduce network traffic.

Fig. 5 schematically illustrates a summary-based caching strategy used in the context of a cooperative replicated cache scheme. In the following, we present an aspect CollaborativeCachePolicy (see Fig. 6) realizing cache groups which replicate data among them as introduced in the previous example, but which also uses summaries to selectively get data from farther caches outside the cache group. These two sets of hosts are represented by groups cacheGroup and summaryHosts, respectively (line 3). Summary information is shared between hosts of the cache groups and the farther hosts at the border using AWED's group sharing feature. This provides for a concise integration of summaries and is appropriate because summary-based caching algorithms only infrequently update summaries. A simpler (and at times more inefficient) sharing mechanism than for the cached data itself can therefore be used for them.

Overall, the aspect consists of a three step remote sequence replPolicy (line 20), which first matches the cache initialization, followed by repeated cache accesses and cache replication operations. An explicit equality test ensures that the put operation in the third step concerns the same cache objects (identified through their keys) as looked up in the second step (assuming that the cache object does not change).

Concretely, at cache initialization time (see the pointcut at line 8) the two host groups are set up as well as initial summary information (advice at line 25). A cache access using getCache (line 11) first looks up the value locally, and, if not found in the cache group attempts to acquire it from the outside. The advice at line 30 first accesses the local cache. If the data is not found, it calls proceed to trigger a synchronous remote advice (line 39) which (because of the on clause) queries hosts of group summaryHosts: this is achieved using a filter selecting hosts whose summaries indicate that the value should be present. The remotely executed advice body returns the query result and requests updates of the summaries if a threshold number of cache modifications has been exceeded (this accounts for the basic property of infrequent updates of summary information, see [18]). The replication within the cache group is achieved as above by matching put operations using the pointcut putCache (line 15). This pointcuts triggers the advice at line 48 which executes a put operation on all hosts in the cache group which are different from the host where the original put occurred.

\subsection{Distribution and Clustering}

In [29], Soares et al. illustrate how AOP techniques can be employed to explicitly introduce distribution within existing, non-distributed applications. For this, AspectJ is employed to automatically insert the required RMI code fragments. Their proposal requires two types of aspects: one aspect for handling server distribution concerns and one aspect for handling client distribution concerns. At the server side, a remote interface is generated for each object that should be distributable and the server-side aspect declares the remote objects to implement these generated interfaces. Additional methods are introduced by means of the server 


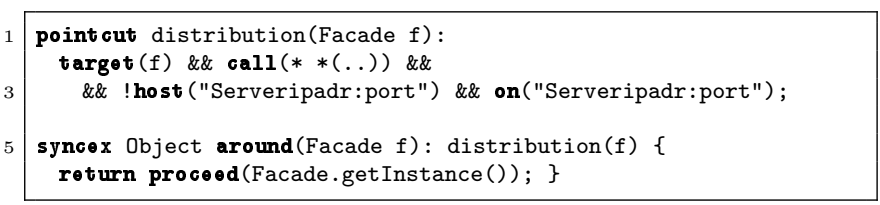

Figure 7: Distribution as an aspect

aspect to implement various technical details to support references to server objects (by default RMI sends a copy of the server object to the client). The client side aspect is responsible for capturing and redirecting the local method calls and declaring these methods to throw remote exceptions. In addition, each method specified in the remote interface requires a dedicated redirection advice, as AspectJ does not allow to change the target object in a proceed statement which is required to redirect the calls to the remote objects.

AWED allows for a more elegant solution, which does not require the overhead of introducing the required RMIspecific code. AWED allows to solve this distribution problem using a single aspect which is illustrated in figure 7 . The distribution pointcut selects all calls to Facade methods on the client and makes sure that the accompanying advice is only executed at the server side. By employing negation of the host designator, calls on the server side will not match the pointcut themselves. The redirection behavior is encapsulated in a synchronous around advice. As the around advice gets executed on the server host, the getInstance method of the Facade class will retrieve an instance which is local to the server host (this could be generalized in order not to rely on a single object.) The proceed expression will invoke the original behavior on that Facade instance located on the server host. The AWED solution improves on the AspectJ-based solution: first, there is no need for RMI specific code to be injected in the server classes, which is a tedious process that is not always possible, and secondly, only one aspect with one pointcut and advice suffices while in the AspectJ solution at least two aspects and a pointcutadvice pairs for each method in the Facade class are necessary. Note that AWED shares this advantage with other middleware-based AOP approaches, such as DJCutter [24] and DAOP [26].

When multiple servers are available to handle remote requests, one can choose to cluster these servers together such that incoming requests can be dispersed, e.g., to balance the server load. Again, AWED provides an elegant solution and allows this clustering behavior to be encapsulated in a single aspect. Figure 8 illustrates this clustering pointcut. All available servers are part of the ServerGroup and the on designator specifies that the accompanying advice should be executed only on a server that is part of that specific group. As only one specific host should be the target of the redirection, a Round Robin load balancing mechanism is employed, which assigns a server host on a rotating base.

\section{IMPLEMENTATION}

Two of the main features the AWED language requires from its underlying middleware implementation are the ability to intercept joinpoints from other hosts and the capability to execute advice on other hosts. A static aspect compiler, as for instance employed by AspectJ [21], is not well

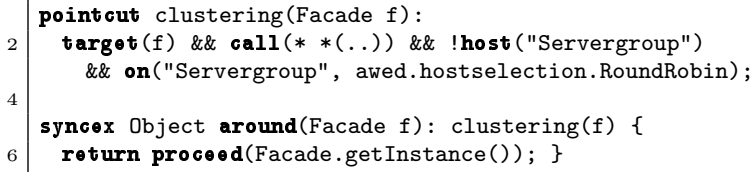

Figure 8: Clustering as an aspect

suited to facilitate a flexible distributed AOP platform, as this setup requires all aspects to be present on all the applicable hosts at compile or weave time. As such, all hosts need to be known and fixed in advance, which removes a lot of flexibility. A dynamic AOP approach however, allows to dynamically add/remove hosts and aspects, which is an important feature for large-scale distributed systems.

Therefore, we have chosen the JAsCo dynamic AOP framework as an implementation platform for the AWED language. JAsCo can be easily extended and provides highly efficient advice execution through its Hotswap and Jutta systems [32]. Furthermore, JAsCo already natively supports a model of stateful aspects based on finite state machines [33], which can be extended to support distributed sequences as well. In the remainder of the paper we present DJAsCo, an extension of the base JAsCo system for explicit distribution. We first briefly introduce the JAsCo run-time architecture and its optimizations. Afterwards, the DJAsCo run-time architecture and our prototype implementation are discussed in detail, and a performance evaluation is presented. The DJAsCo extension has been made publicly available as a part of the regular JAsCo distribution [20].

\subsection{JAsCo run-time infrastructure}

The JAsCo run-time infrastructure is based on a central connector registry that manages the registered connectors ${ }^{2}$ and aspects at run-time (see figure 9). This connector registry serves as the main addressing point for all JAsCo entities and contains a database of connectors and instantiated aspects. Whenever a connector is loaded into or removed from the system at run-time, the connector registry is notified and its database of registered connectors and aspects is automatically updated. The left-hand side of Figure 9 illustrates a JAsCo-enabled class from which the joinpoint shadows are equipped with traps. As a result, whenever a joinpoint is triggered, its execution is deferred to the connector registry, which looks up all connectors that are registered for that particular joinpoint. The connector on its turn dispatches to the applicable aspects.

The connector registry has an open plugin-based architecture that allows to easily extend the joinpoint interception, aspect lookup (pointcut evaluation) and advice execution parts, in particular for composition and optimization purposes.

In addition to the connector registry, the run-time architecture consists of two other systems: HotSwap and Jutta. HotSwap allows to dynamically install traps only at those joinpoint shadows that are subject to aspect application. When a new aspect is deployed, the applicable joinpoints

\footnotetext{
${ }^{2} \mathrm{JAsCo}$ introduces explicit connectors that instantiate and deploy aspects onto a concrete (component) context. In our AspectJ-based language, the aspect construct is responsible for both. An AspectJ-like aspect can easily map onto a connector-aspect pair in JAsCo.
} 


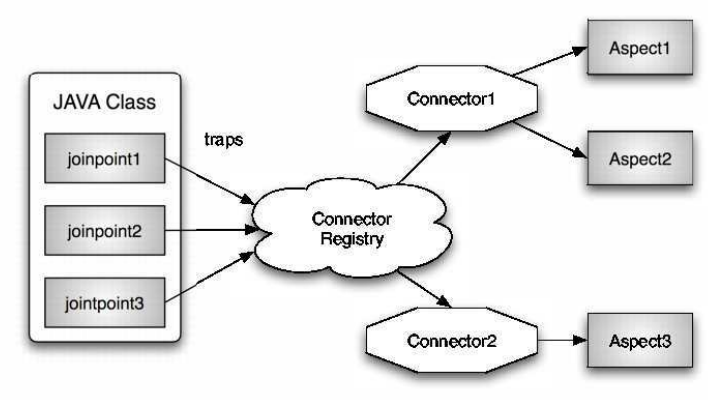

Figure 9: JAsCo run-time architecture

shadows are hot-swapped at run-time with their trapped equivalents. Likewise, the original byte code is reinstalled when an aspect is removed and no other aspects are applicable on the joinpoint shadow at hand. Jutta on the other hand, is a just-in-time compiler for aspects that allows to generate a highly optimal code fragment for every joinpoint shadow. By caching these code fragments, an important performance gain is realized. The current version of the JAsCo run-time weaver, based on HotSwap and Jutta, is able to compete performance-wise with statically compiled aspect languages such as AspectJ, while still preserving dynamic AOP features [13]. A major concern of the DJAsCo design is to preserve compatibility of the weaver with these two tools, in particular to enable the optimization of remote pointcuts.

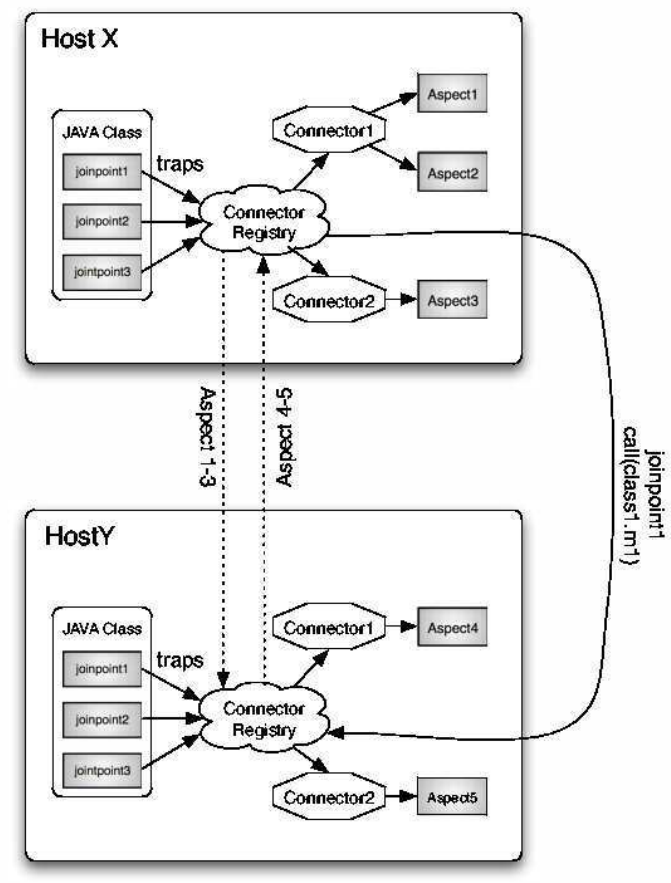

Figure 10: DJAsCo distributed run-time architecture. The aspects are distributed to all relevant hosts. Joinpoint1 occurs in Host $X$ and is also sent to Host $Y$ in order to trigger aspects on that remote joinpoint.

\subsection{DJAsCo run-time architecture}

The JAsCo run-time architecture can be distributed using two different strategies: either a single connector registry is kept for all hosts or each host separately maintains a dedicated connector registry. The first solution has the advantage that a single registry is responsible for the aspect execution, whereas the second solution requires the distributed connector registries to be synchronized. In general, a central entity is considered to be a problematic solution in a distributed setting, as it inherently does not scale and can become a performance bottleneck. Therefore we choose to deploy a separate connector registry at each host (see figure 10).

Every connector registry is responsible for the locally intercepted joinpoints and its locally deployed aspects. In order to allow aspect execution on remote joinpoints, the intercepted joinpoints need to be sent to the other hosts. Likewise, in order to allow aspect execution on remote hosts, the aspects need to be distributed as well. The following sections explain these issues in more detail.

\subsubsection{Remote pointcuts}

In order to execute advice that trigger on remote joinpoints, the joinpoint information should be distributed to all interested hosts. To this end, a plugin for the connector registry has been implemented that: 1) intercepts all joinpoints, 2) prepares them for transmission and 3) sends them to the remote hosts. Joinpoints need to be prepared before transmission as not all joinpoint information might be transmittable. Our current system uses Java Serialization to transmit objects from one host to another. For this, the joinpoint is first stripped from all contextual information (e.g. callee, actual arguments) that is nor serializable nor primitive. Furthermore, the advice implementation cannot access advice variables nor reflectively query joinpoint information that is not serializable. Although this is an important limitation, it is typical in distributed environments. For instance, arguments of Java RMI method invocations need to be serializable or primitive as well.

In a last step, the joinpoint information is sent to the remote hosts. In order to locate and send this information to other interested hosts, the JGroups framework is employed [6]. JGroups is a well-known toolkit for reliable multicast communication. In addition, JGroups supports a wide range of network protocols, which makes our system independent of specific network technologies.

JAsCo's stateful aspects, i.e., finite-state based sequences [33], have been extended to a distributed setting in order to implement AWED's distributed sequences. This is a rather straightforward process, because the state of a sequence pointcut is not managed by the JAsCo run-time infrastructure (deployed locally, see figure 10), but by the aspect itself. The aspect intercepts remote joinpoints, matching its stateful pointcut description in a similar way as for joinpoints matching regular pointcuts. Afterwards, the internal state is updated by firing the relevant transition(s) in the internal state machine.

\subsubsection{Aspect Distribution}

In order to execute advice on remote hosts, the aspects themselves should also be distributed to the host(s) in question. One solution would be to force an administrator to manually deploy the aspects on every applicable host. How- 
ever, as an advice execution host sometimes depend on complex expressions with several variables, it might be difficult to manually deploy the aspects onto remote hosts in an optimal fashion. For instance, deploying aspects to hosts where they can never be applicable is useless and wastes the system resources of those particular hosts. Hence, the DJAsCo extension automatically distributes the aspects to all remote hosts that might be applicable. When a new host joins, the DJAsCo run-time infrastructure detects this event, and the host automatically receives the possibly applicable aspects. Likewise, when new aspects are deployed at a particular host, they are automatically deployed at the relevant remote hosts. It is possible to avoid this automatic deployment of aspects on remote hosts by marking them with the single modifier.

Technically, JGroups is again employed to transfer the aspects and to be informed of changes in the network setup such as newly joined hosts. In contrast to joinpoints, aspects are class-based entities and it suffices to send the class byte-code to the remote hosts. Hence, possible serialization problems are avoided.

\subsubsection{Synchronous Advice Execution}

The default mode for advice execution is asynchronous with respect to advice executions on remote hosts. However, when an advice is marked with the syncex modifier, it needs to be executed synchronously, i.e., the host where the joinpoint occurs, waits for this advice to be executed. As such, the specified aspect precedence on the joinpoint host is still guaranteed. The return value of the around advice is sent back to the original joinpoint host and processed in the regular around advice chain. A proceed to the original behavior is however still a local proceed (likewise to proceed in an asynchronous advice), which means that the joinpoint proceeds on the host where the advice is executed and not on the joinpoint host.

DJAsCo implements a synchronous advice execution by generating a proxy aspect at the joinpoint host. This proxy aspect is automatically generated and dynamically weaved when an aspect, defining a synchronous advice execution, is deployed. The proxy aspect's advice delegates to the appropriate execution host where the original advice is executed. The JGroups framework is again employed for the synchronous communication between the involved hosts.

\subsubsection{State Sharing}

The AWED language supports state sharing between different instances of the same aspect type regardless of the location and/or VM where they are executed. In order to implement local sharing, DJAsCo generates one master field on every host for each locally shared aspect field. All aspect instances of the aspect type on that host automatically refer to that field using Java RMI. Field queries and updates are automatically redirected to the shared field. This redirection takes place by employing another AWED aspect that is dynamically generated and weaved when an aspect, defining a shared field, is being deployed. Visibility modifiers for the fields (such as private) do not hinder the sharing implementation because they can be overridden at run-time.

The global state sharing could be implemented in a similar fashion, i.e., having one globally shared field. However, this solution suffers from a serious robustness problem as all aspect instances of the same type would rely on one specific

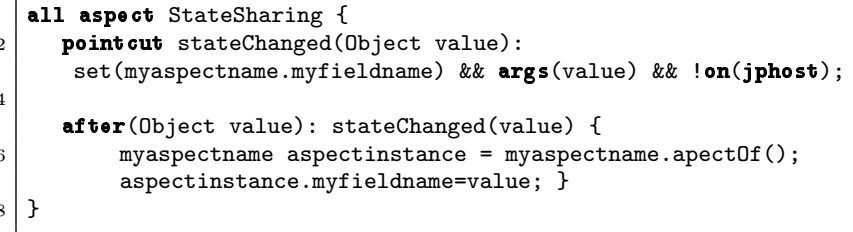

Figure 11: State sharing as a AWED aspect

host that holds the shared field. Therefore, the local master fields are explicitly synchronized using yet another AWED aspect that is automatically generated at deployment time. Figure 11 illustrates a simplified version ${ }^{3}$ of this global state sharing aspect. The after advice is triggered for every state change of the myfieldname field of the myaspectname aspect. The advice is executed on every host except on the one that triggered the joinpoint. As such, the state change is propagated to all other hosts. The advice implementation first fetches an aspect instance of the given type on the host where it is executing and than changes the value to the newly assigned value. Because all aspect instances on that host refer to the same field, the new value is immediately propagated to all aspect instances of the myaspectname type on the host at hand.

\subsubsection{Optimization of remote pointcuts}

The JAsCo HotSwap and Jutta systems are compatible with the DJAsCo architecture. Because all aspects are present at every applicable host (even aspects that might execute their advice elsewhere), the local HotSwap system still knows where to insert traps. Aspects that do not define pointcuts relevant for the local host are not deployed and are of no interest to the HotSwap system as they do not induce newly trapped joinpoints. Remote joinpoints are represented similarly to local joinpoints. Hence, the Jutta system is still able to generate and cache a code fragment for executing the joinpoint locally. As such, apart from the network delay and serialization/deserialization cost, no additional overhead is required for remote pointcuts and distributed advice executions.

\subsection{Evaluation}

To conclude this section we present results on two evaluations using aspects with explicit distribution for refactoring and extension of the standard replication strategy of JBoss.

\subsubsection{Refactoring of JBoss replication code}

In the motivation we have identified replication and transaction as two functionalities contributing to crosscutting within JBoss cache. Transaction handling in JBoss Cache is basically done using a two phase commit protocol. First the transaction is executed locally, requiring, in particular, only the acquisition of local locks. Once the commit method is invoked in the current transaction, all the modifications are replicated to all the nodes in a cache replication group: at remote hosts a prepare phase is executed and, if successful in all nodes, the transaction is committed. If a prepare phase in any node fails, the transaction is rolled back at all nodes.

${ }^{3}$ Notice that this aspect has been simplified for presentation purposes. For example, it does not cope with the fact that aspect types might not be present on every host. 


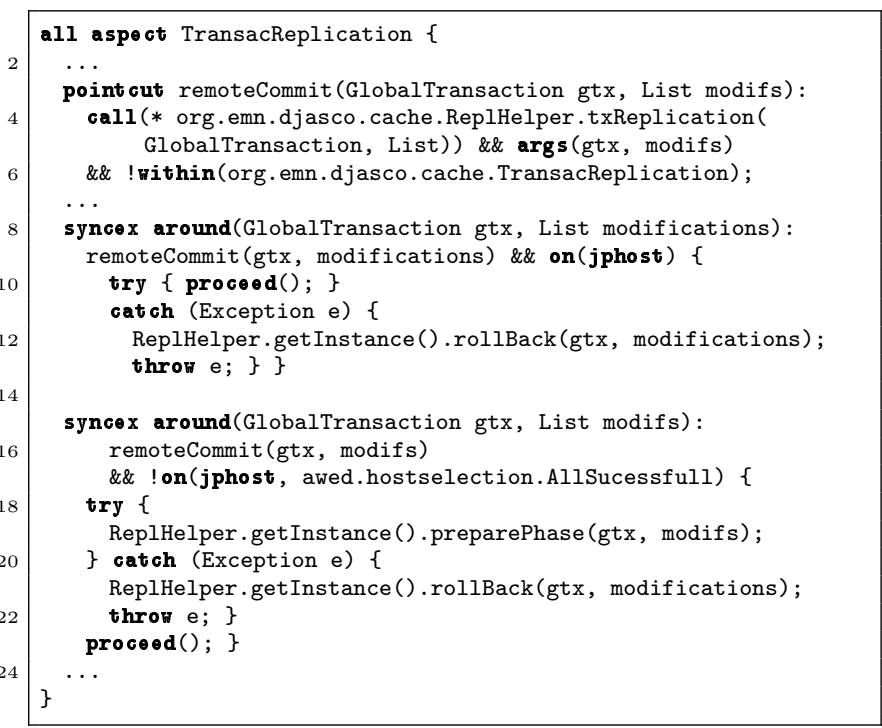

Figure 12: Refactoring the JBoss replication code

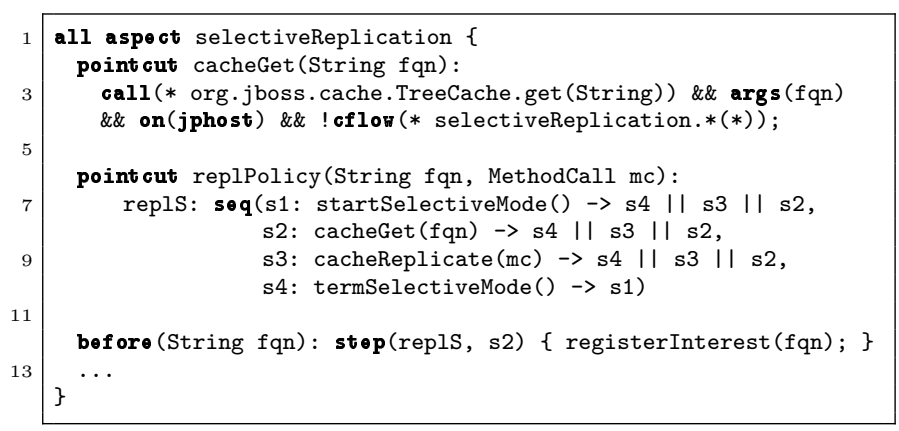

\section{Figure 13: Extending the JBoss replication code}

Figure 12 shows (essential parts of) a re-implementation of the transaction-guarded replication strategy of JBoss Cache. AWED allows to cleanly modularize the transaction-guarded replication protocol in a single aspect. The first advice realizes the protocol part of the host that initiates the transaction: it handles data within the local cache through a call to proceed and if something fails it rolls the transaction back. The second advice executes replication handling at other nodes through a remote call to preparePhase() and raises an exception if something fails (the rollback functionality for the remote hosts is implemented by another advice not shown in the example).

\subsubsection{Extension of the JBoss replication strategy}

As a second evaluation, we realized an extension to the JBoss standard replication strategy: data should only be replicated to nodes that have explicitly requested it, i.e., new objects inserted in a cache group are not replicated spontaneously.

Figure 13 shows the main parts of an appropriate aspect definition using AWED (the complete implementation, approx. twice as large, is detailed in [9]). The implementation uses a sequence pointcut repls which implements a distributed protocol: this protocol starts selective replica- tion mode, followed by interception of get operations (which explicitly indicate interest in some data) and cache replication operations, until a selective mode termination method is called. Data which is to be replicated is selected through the advice applied before get operations (step 2 in the sequence replS) which registers interest to the data.

\subsubsection{Comparison to a JBoss-only solution}

To conclude this evaluation, let us compare the two previous examples to an implementation based only on JBoss. JBoss Cache implements transaction replication using three main classes TreeCache, ReplicationInterceptor and SynchronizationHandler. Reflection is used to (un)marshal messages sent between nodes in the cluster. This architecture is augmented with a before-completion and aftercompletion idiom that is present in different classes. Methods include code to hold a special state, which is used to coordinate protocol stages. Tracing the default replication behavior and integration of the proposed algorithm is far from trivial due to the crosscutting these functionalities are subject to.

Contrary to the tangled implementation of transaction and replication code in JBoss Cache, our aspect refactoring clearly separates replication and transactions (transactions appear, apart from their setup, only in exception handlers). Concretely, we have been able to refactor the scattered replication functionality and the corresponding transaction handling which amounts to around 500 LOC in JBoss into one aspect of around 100 LOC. Furthermore, our aspect does not require any particular transaction management but reuses the default transaction management of JBoss Cache. Finally, the second example shows how extensions can be easily integrated using AWED, in particular, because distributed protocols can concisely be expressed using sequence aspects.

\section{RELATED WORK}

D [34] has been the first aspect language with means for explicit distribution. This approach includes, in particular, COOL, a synchronization sublanguage, and RIDL, a sublanguage for the definition of remote interfaces. The former essentially supports the declarative definition of mutual exclusion relations between objects. The latter allows to specify the semantics of remote method invocation between different execution spaces, in particular the argument passing semantics. However, D does not provide equivalents for most features of AWED, in particular, remote sequence aspects, distributed advice and distributed aspects.

JAC [25] is a dynamic AOP-framework, which, in contrast with other AOP approaches, does not introduce a dedicated aspect language, but describes aspects in terms of regular OO-abstractions. Their proposed framework is extended with the notion of a distributed pointcut definition. This pointcut definition extends a regular pointcut with the ability to specify a named host that delimits the context in which the joinpoint should be detected. In contrast with AWED, JAC does not provide support to delimit the context in which distributed advices should be executed, nor does it support remote sequences and instantiable distributed aspects. In order to manage the distributed deployment of aspects, JAC replicates its Aspect-Component manager, which is similar to the DJAsCo connector registry, on the involved remote hosts. A consistency protocol makes sure 
that whenever aspects are weaved at one specific host, the same aspects are also weaved at the other involved hosts.

DJcutter [24] is an extension of the AspectJ language, which extends a subset of AspectJ's constructs to make them behave as remote pointcuts. Similar to JAC and AWED, DJcutter introduces an explicit host pointcut designator that allows to delimit the context in which joinpoints should be detected. In addition, DJcutter supports distributed cflow pointcuts if the base application is implemented using a custom socket implementation. Similar to AWED, the state information of a joinpoint is exposed to an advice by using the args and target designators, from which by default, the values are remotely transferred by copy. At the runtime level, DJcutter proposes a centralized aspect-server. This server gathers joinpoint information of remote pointcut definitions and executes the related advices local to the aspect-server. The advice server constitutes a bottleneck in a large distributed systems and makes the implementation of, e.g., cache replication much more complex compared to AWED, because the aspect implementer is responsible to manage and execute the appropriate methods on the distributed hosts.

There are some approaches which have essentially developed for the sequential case but provide some mechanisms for AOP in distributed environments. CaesarJ [23] and PROSE [27] are part of this class of approaches. They mainly provide a sequential aspect model based on collaborations between aspects and classes but allow aspects to be created on remote hosts. Concretely, CaesarJ allows the host where an aspect is to be executed to be explicitly specified when the aspect is created. In contrast to our approach no further means to explicitly refer to remote aspect interactions are provided: they do not provide mechanisms for sharing of aspect state, management of distributed aspect instances as well as distributed advice execution.

Another important category of approaches applying AOP to distributed applications, e.g., JBoss AOP [1], Spring AOP [3] and several research approaches, such as that proposed by Duclos et al. [17], essentially allow non-distributed aspects to manipulate applications implemented using an existing framework for distribution. While they integrate some features which can be found in our system (such as dynamic aspect application), they do not provide features for explicit specification of distribution issues in aspects. In contrast to our approach, replicating data in remote caches can thus only be done, for instance, by an aspect which repeatedly calls existing methods for put operations on remote caches. Similarly, Cohen and Gil [11] essentially preserve a non-sequential AOP framework. They however propose an extension to directly express aspects involving J2EE-based crosscutting concerns between servers and clients through so-called tier-cutting concerns using a remotecall construct. They do not support any of our more advanced concepts, such as remote sequences, distributed advice and distributed aspects. Taking an application-oriented view, Colyer and Clement [12] investigate the benefits of using AspectJ, also a sequential AOP approach, to modify interfaces of Websphere, IBM's J2EE-based application server. All of these approaches (as well as several AO frameworks for distributed middleware, such as DAOP [26] and Lasagne [31]) do not propose features for explicit distribution in the aspect language, which are useful, as we have shown in this paper, also for EJBs and similar frameworks.
Finally, there are some AOP approaches which investigate concurrency issues and thus are also directly relevant to some of the distribution issues we have considered. To cite one example, Andrews [5] considers aspect weaving in a process calculus relying on asynchronous communication, essentially by aggregating fine-grained concurrent executions into coarser-grained ones. While this technique should be in principle applicable to distributed aspect languages, his work does not provide explicit means to support distributionspecific concerns, such as data replication.

\section{CONCLUSION AND FUTURE WORK}

In this paper we have provided evidence that current systems for AOP with explicit distribution are limited. We presented AWED, a language including distributed sequence pointcuts, remote advice execution, and distributed advice. We also introduced DJAsCo, an implementation of AWED based on the JAsCo AOP system. Finally, we provided evidence that our concepts of explicit distribution in aspects can be usefully applied to cache replication problems.

At the language level, AWED constitutes a first proposal for a comprehensive aspect language for explicit distribution. However, a number of issues remain to be explored, in particular, integrated means for synchronization of asynchronous advice and finer-grained control of aspect deployment, instantiation and data sharing. The AWED implementation as a JAsCo extension should be improved by performance optimizations specific for the distributed context.

Acknowledgements. We thank Jean-Marc Menaud for our fruitful discussions on replicated caches and the anonymous reviewers for their valuable remarks.

\section{REFERENCES}

[1] JBoss AOP. http://jboss.com/products/aop.

[2] JBoss home page. http://jboss.com.

[3] Spring AOP. http://www.springframework.org/.

[4] C. Allan et al. Adding trace matching with free variables to AspectJ. In R. P. Gabriel, editor, Proc. of OOPSLA'05. ACM Press, Oct. 2005.

[5] J. H. Andrews. Process-algebraic foundations of aspect-oriented programming. In Proc. of the 3rd International Conference on Metalevel Architectures and Separation of Crosscutting Concerns, volume 2192 of $L N C S$, pages 187-209, 2001.

[6] B. Ban. JGroups, a toolkit for reliable multicast communication. http://www.jgroups.org/, 2002.

[7] B. Ban and B. Wang. JBossCache Reference Manual V. 1.2. JBoss Inc., 2005.

[8] G. Barish and K. Obraczka. World wide web caching: Trends and techniques. IEEE Communications Magazine, May 2000.

[9] D. Benavides Navarro, M. Südholt, W. Vanderperren, B. De Fraine, and D. Suvée. Explicitly distributed AOP using AWED. Technical report, INRIA, Feb. 2006.

[10] S. Bouchenak, A. Cox, S. Dropsho, S. Mittal, and W. Zwaenepoel. AOP-based caching of dynamic web content: Experience with J2EE applications. Technical Report RR-5483, INRIA, 2005.

[11] T. Cohen and J. Gil. AspectJ2EE = AOP + J2EE: Towards an aspect based, programmable and 
extensible middleware framework. In Proc. ECOOP '04, volume 3086 of LNCS. Springer-Verlag, 2004.

[12] A. Colyer and A. Clement. Large-scale AOSD for middleware. In Proc. of AOSD'04. ACM Press, 2004.

[13] B. De Fraine, W. Vanderperren, D. Suvée, and J. Brichau. Jumping aspects revisited. In R. E. Filman, M. Haupt, and R. Hirschfeld, editors, Dynamic Aspects Workshop, pages 77-86, Mar. 2005.

[14] R. Douence, P. Fradet, and M. Südholt. A framework for the detection and resolution of aspect interactions. In Proceedings of GPCE'02, volume 2487 of $L N C S$, pages 173-188. Springer-Verlag, Oct. 2002.

[15] R. Douence, P. Fradet, and M. Südholt. Composition, reuse and interaction analysis of stateful aspects. In Proc. AOSD'04. ACM Press, Mar. 2004.

[16] R. Douence, T. Fritz, N. Loriant, J.-M. Menaud, M. Ségura-Devillechaise, and M. Südholt. An expressive aspect language for system applications with arachne. In Proc. AOSD'05. ACM Press, Mar. 2005.

[17] F. Duclos, J. Estublier, and P. Morat. Describing and using non functional aspects in component based applications. In Proc. of AOSD'02, pages $65-75$. ACM Press, 2002.

[18] L. Fan, P. Cao, J. M. Almeida, and A. Z. Broder. Summary cache: a scalable wide-area web cache sharing protocol. IEEE/ACM Transactions on Networking, 8(3):281-293, 2000.

[19] ICP. Internet Cache Protocol. http://icp.ircache.net/.

[20] JAsCo. JAsCo website. http://ssel.vub.ac.be/jasco/.

[21] G. Kiczales, E. Hilsdale, J. Hugunin, M. Kersten, J. Palm, and W. G. Griswold. An overview of AspectJ. In J. L. Knudsen, editor, Proc. ECOOP 2001, LNCS 2072, pages 327-353, Berlin, June 2001. Springer-Verlag.

[22] G. Kiczales, J. Lamping, A. Mendhekar, et al. Aspect-oriented programming. In M. Akşit and S. Matsuoka, editors, Proc. ECOOP 1997, volume 1241 of $L N C S$, pages 220-242. Springer Verlag, 1997.

[23] M. Mezini and K. Ostermann. Variability management with feature-oriented programming and aspects. In Proc. ESEC/FSE'04, pages 127-136, 2004.

[24] M. Nishizawa, S. Shiba, and M. Tatsubori. Remote pointcut - a language construct for distributed AOP. In Proc. of AOSD'04. ACM Press, 2004.

[25] R. Pawlak, L. Seinturier, L. Duchien, and G. Florin. JAC: A flexible solution for aspect-oriented programming in Java. In Proceedings of Reflection'01, volume 2192 of $L N C S$. Springer-Verlag, Sept. 2001.

[26] M. Pinto, L. Fuentes, M. Fayad, and J. Troya. Separation of coordination in a dynamic aspect oriented framework. In Proc. of AOSD'02. ACM Press, 2002. short paper.

[27] A. Popovici, T. Gross, and G. Alonso. Dynamic weaving for aspect-oriented programming. In G. Kiczales, editor, Proc. AOSD 2002, pages 141-147. ACM Press, Apr. 2002.

[28] M. Ségura-Devillechaise, J.-M. Menaud, G. Muller, and J. L. Lawall. Web cache prefetching as an aspect. In Proc. of AOSD'03. ACM Press, 2003.

[29] S. Soares, E. Laureano, and P. Borba. Implementing distribution and persistence aspects with AspectJ. In Proceedings of OOPSLA'02, pages 174-190. ACM Press, 2002.

[30] D. Suvée and W. Vanderperren. JAsCo: An aspect-oriented approach tailored for component based software development. In M. Akşit, editor, Proc. AOSD 2003, pages 21-29. ACM Press, Mar. 2003.

[31] E. Truyen et al. Dynamic and selective combination of extensions in component-based applications. In Proc. ICSE 2003, May 2001.

[32] W. Vanderperren and D. Suvée. Optimizing JAsCo dynamic AOP through HotSwap and Jutta. In R. Filman, M. Haupt, K. Mehner, and M. Mezini, editors, DAW: Dynamic Aspects Workshop, pages 120-134, Mar. 2004.

[33] W. Vanderperren, D. Suvee, M. A. Cibran, and B. De Fraine. Stateful aspects in JAsCo. In Proc. of Software Composition (SC'05), volume 3628 of LNCS. Springer-Verlag, Apr. 2005.

[34] C. Videira Lopes. D: A Language Framework for Distributed Programming. PhD thesis, College of Computer Science, Northeastern University, 1997. 